\title{
BOUNDED SOLUTIONS OF SOME ABSTRACT DIFFERENTIAL EQUATIONS
}

\section{S. ZAIDMAN ${ }^{1}$}

Introduction. We give here two theorems abstract versions and generalization of some results in our previous papers [2], [3]. ${ }^{2}$ For semigroup theory applied here look at [1].

THEOREM 1. Let $T(t)$ be a strongly continuous semigroup in the Banach space $X$ such that $T(o)=I$ and $\lim _{t \rightarrow \infty} T(t) x=0 \quad \forall x \in X$.

Let $A$ be its infinitesimal generator and suppose $\exists \lambda_{0} \in C$ such that $\left(\lambda_{0}-A\right)^{-1}$ is a compact operator in $X$.

Let $u(t) \in C^{1}(-\infty, \infty ; X), u(t) \in D(A) \forall t \in(-\infty, \infty)$, be a solution of the abstract differential equation

$$
u^{\prime}(t)=A u(t)
$$

which is defined for all $t \in(-\infty, \infty)$.

Suppose $\exists c>0$ such that $\int_{t}^{t+1}\|u(t)\|_{X}^{2} d t \leqq c, \forall t \in(-\infty, \infty)$. Then $u(t) \equiv \theta, \quad \forall t \in(-\infty, \infty)$.

ProOF OF THE THEOREM. We remark first that from our hypothesis

$$
\sup _{-\infty<t<\infty} \int_{t}^{t+1}\|u(t)\|_{X}^{2} d t<\infty
$$

there follows the existence of a sequence $\left(t_{n}\right)_{1}^{\infty} \downarrow-\infty$, such that $\sup _{n \in N}\left\|u\left(t_{n}\right)\right\| x<\infty$. We remark also that, for each $t \geqq t_{0}$, the function $u(t)$ admits the representation $u(t)=T\left(t-t_{0}\right) u\left(t_{0}\right)$.

Also, a well-known fact is that $\left(\lambda_{0}-A\right)^{-1} T(t)=T(t)\left(\lambda_{0}-A\right)^{-1}$. Let us put now: $\omega(t)=\left(\lambda_{0}-A\right)^{-1} u(t)$; obviously sequence $\left\{\omega\left(t_{n}\right)\right\}_{1}^{\infty}$ contains a convergent subsequence $\omega\left(t_{n_{k}}\right) \rightarrow \omega_{\infty} \in X$, and $\omega(t)=T\left(t-t_{0}\right) \omega\left(t_{0}\right)$, $\forall t \geqq t_{0}$. Let $t \in(-\infty, \infty)$ be fixed and $k_{0}$ big enough to get $t_{n_{k}}<t$ for $k \geqq k_{0}$. Then we obtain, as $t>t_{n_{k}}$,

$$
\omega(t)=T\left(t-t_{n_{k}}\right) \omega\left(t_{n_{k}}\right)=T\left(t-t_{n_{k}}\right)\left[\omega\left(t_{n_{k}}\right)-\omega_{\infty}\right]+T\left(t-t_{n_{k}}\right) \omega_{\infty}
$$

and, consequently,

Received by the editors February 17, 1969.

1 Supported by the N.R.C. of Canada.

2 We thank the referee whose report helped us to improve the presentation of Theorem 1 and the original Theorem 2. 


$$
\begin{aligned}
\|\omega(t)\| & \leqq\left\|T\left(t-t_{n_{k}}\right)\right\|_{\mathfrak{L}_{(X, X)}}\left\|\omega\left(t_{n_{k}}\right)-\omega_{\infty}\right\|_{X}+\left\|T\left(t-t_{n_{k}}\right) \omega_{\infty}\right\|_{X} \\
& \leqq L\left\|\omega\left(t_{n_{k}}\right)-\omega_{\infty}\right\|_{X}+\left\|T\left(t-t_{n_{k}}\right) \omega_{\infty}\right\|_{X}, \quad \forall k \geqq k_{0} .
\end{aligned}
$$

(Because of the strong continuity of $T(t)$ and asymptotic decay to 0 , it follows that $\|T(t)\|_{\mathfrak{L}} \leqq L$.) This obviously implies $\omega(t)=\theta$, hence $u(t)=\theta$.

In the second result, conditions on the compactness of $\left(\lambda_{0}-A\right)^{-1}$ and on strong asymptotic decay of $T(t)$ are replaced by the condition of strong asymptotic decay of the adjoint semigroup $T^{*}(t)$. Precisely, we have

TheOREM 2. Let $T(t)$ be a strongly continuous semigroup in the Banach space $X$, and let us suppose that

$$
\lim _{t \rightarrow \infty} T^{*}(t) x^{*}=0, \quad \forall x^{*} \in X^{*}
$$

$T^{*}(t)$ being, $\forall t \in(0, \infty)$, the adjoint operator of $T(t)$ acting in the dual space $X^{*}$. Let $A$ be the infinitesimal generator of $T(t)$ and $u(t) ;-\infty<t$ $<\infty \Rightarrow D(A)$ be a strong solution of the differential equation $u^{\prime}(t)=A u(t)$. Suppose $\exists c>0$ such that

$$
\int_{t}^{t+1}\|u(t)\|_{X}^{2} d t \leqq c, \quad-\infty<t<\infty .
$$

Then $u(t) \equiv \theta,-\infty<t<\infty$.

Proof of theorem 2. As in Theorem 1 we find a sequence $\left(t_{n}\right)_{1}^{\infty}$ of real numbers, such that $\lim _{n \rightarrow \infty} t_{n}=-\infty$ and $\left\|u\left(t_{n}\right)\right\|_{x} \leqq M$, $n=1,2, \cdots$. For arbitrary real $t_{0}$, we will have $t_{n}<t_{0}$ if $n \geqq n_{0}$. Then we may represent $u\left(t_{0}\right)$ as: $u\left(t_{0}\right)=T\left(t_{0}-t_{n}\right) u\left(t_{n}\right)$. Take now an arbitrary $x^{*} \in X^{*}$; then, if $\langle$,$\rangle means duality between X$ and $X^{*}$ we will have $\left\langle x^{*}, u\left(t_{0}\right)\right\rangle=\left\langle x^{*}, T\left(t_{0}-t_{n}\right) u\left(t_{n}\right)\right\rangle=\left\langle T^{*}\left(t_{0}-t_{n}\right) x^{*}, u\left(t_{n}\right)\right\rangle$ and

$$
\left|\left\langle x^{*}, u\left(t_{0}\right)\right\rangle\right| \leqq\left\|T^{*}\left(t_{0}-t_{n}\right) x^{*}\right\|\left\|u\left(t_{n}\right)\right\| \leqq M\left\|T^{*}\left(t_{0}-t_{n}\right) x^{*}\right\| .
$$

As $n \rightarrow \infty, T^{*}\left(t_{0}-t_{n}\right) x^{*} \rightarrow \theta$; hence $\left\langle x^{*}, u\left(t_{0}\right)\right\rangle=\theta \forall x^{*} \in X^{*}$ and consequently $u\left(t_{0}\right)=\theta$.

Remark. An example of the situation arising in Theorem 2 is the following: (see our paper [3]).

Take $X=L^{2}\left(R^{n}\right) ; T(t) f=e^{-\left(8_{1}^{2}+\cdots+s_{n}^{2}\right) t} f(s)_{t \geq 0}, \quad s=\left(s_{1} \cdots s_{n}\right), f(s)$ $\in L^{2}\left(R^{n}\right)$. It is immediate that $T^{*}(t)=T(t), \forall t \geqq 0$, and that

$$
\lim _{t \rightarrow \infty}\left(\int_{R^{n}} e^{-2\left(s_{1}^{2}+\cdots+s_{n}^{2}\right) t} f^{2}(s) d s\right)^{1 / 2}=0, \quad \forall f(s) \in L^{2} .
$$


Also, $A f=-\left(s_{1}^{2}+\cdots+s_{n}^{2}\right) f(s)$ with $D(A)=\left\{f \in L^{2},-|s|^{2} f \in L^{2}\right\}$. Hence our Theorem 2 applies in this case.

\section{References}

1. E. Hille and R. S. Phillips, Functional analysis and semigroups, Amer. Math. Soc. Colloq. Publ., Providence, R. I., 1957.

2. C. Foiaş and S. Zaidman, Almost-periodic solutions of parabolic systems, Ann. Scuola. Norm. Sup. Pisa, 1962.

3. S. Zaidman, Soluzioni limitate e quasi-periodiche dell'equazione del calore nonomogenea. I, Rend. Accad. Naz. Lincei, 1961.

UNiversité de MONTRÉAL 